\title{
riccafd
}

Revista Iberoamericana de Ciencias de la Actividad Física y el Deporte

\section{CONOCIMIENTO PEDAGÓGICO DEL CONTENIDO: UN ESTUDIO CUALITATIVO EN EL PROFESORADO DE EDUCACIÓN FÍSICA}

\section{PEDAGOGICAL CONTENT KNOWLEDGE: A QUALITATIVE STUDY ON PHYSICAL EDUCATION TEACHER}

Alejandro Almonacid-Fierro', Eugenio Merellano-Navarro², Sebastian Feu Molina ${ }^{3}$, Manuel Vizuete Carrizosa ${ }^{4}$

${ }_{1}^{1}$ Alejandro Almonacid-Fierro. Dr. Actividades Físicas y Artísticas. Facultad de Educación Universidad Católica del Maule, Talca, Chile. aalmonacid@ucm.cl.

${ }^{2}$ Eugenio Merellano-Navarro. Dr. Actividades Físicas y Artísticas. Facultad de Educación Universidad Autónoma de Chile, Talca, Chile; emerellanon@uautonoma.cl

${ }^{3}$ Sebastián Feu Molina. Dr. Actividad Física y el Deporte. Facultad de Educación, Universidad de Extremadura, Badajoz, España. sfeu@unex.es;

${ }^{4}$ Manuel Vizuete Carrizosa. Dr. Historia Contemporánea. Facultad de Educación, Universidad de Extremadura, Badajoz, España. mvizuete@unex.es

Código UNESCO: 580207 Formación Profesional

Clasificación Consejo de Europa: 5 Didáctica y Metodología

DOI:http://dx.doi.org/10.24310/riccafd.2018.v7i3.5521

\section{RESUMEN}

La presente investigación tiene como propósito describir y categorizar el discurso del profesorado de Educación Física con respecto al Conocimiento Pedagógico del Contenido (CPC) en el aula de Educación Física y, por otro lado, develar las comprensiones subjetivas con que los profesores estructuran y organizan sus estrategias didácticas para favorecer el aprendizaje de los estudiantes. En términos metodológicos el estudio es de carácter cualitativo, toda vez que se interpreta el discurso del profesorado en base a la teoría fundamentada, vale decir el procesamiento se sustenta en la deconstrucción de los datos, la comparación constante y la reconstrucción de datos. A partir del proceso de codificación y categorización emergen las principales categorías primarias: conocimiento y aprendizaje, conocimiento del currículo en Educación Física y conocimiento de estrategias didácticas, cuestión que nos permite comprender como los profesores comprenden y despliegan el aprendizaje en el área.

Palabras Clave: educación física, técnica didáctica, conocimiento, formación de profesores (Tesauro UNESCO).

\section{ABSTRACT}

This research aims at describing and categorizing the discourse of Physical Education teachers with regard to Pedagogical Content Knowledge (PCK) in the 
Physical Education classroom and, on the other hand, to reveal the subjective understandings with which teachers structure and organize their didactic strategies to promote student learning. In methodological terms, the study is qualitative in nature, since teachers' discourse is interpreted on the basis of grounded theory, that is to say, processing is based on deconstruction of data, constant comparison and reconstruction of data. From the process of coding and categorization, the main primary categories emerge: knowledge and learning, knowledge of the curriculum in Physical Education and knowledge of teaching strategies, an issue that allows us to understand how teachers understand and deploy learning in the area.

Key Words: Physical education, Classroom techniques, Knowledge, Teacher education (Thesauri UNESCO).

\section{INTRODUCCION}

El Conocimiento Pedagógico del Contenido o Conocimiento Didáctico del Contenido (CDC) como se le conoce en diferentes países, se refiere a la forma en que el docente define los enfoques que utiliza para ajustar el contenido y sus elementos organizados para una mejor enseñanza, vale decir, existe una especial preocupación en comprender la complejidad que significa la transformación del saber pedagógico o especifico del contenido, en aprendizajes de carácter significativo para el estudiante, teniendo en consideración aspectos tales como las técnicas pedagógicas, los conocimientos previos de los alumnos, las concepciones del profesorado y las teorías epistemológicas (1-10).

A partir de los planteamientos de Lee Shulman, a fines de la década del 80 surge el término CDC. Shulman $(8,9,11)$ es un firme defensor de la profesión docente, de su valorización y reconocimiento social, por lo que se empeñó en establecer un cuerpo de conocimientos específicos de la profesión docente, tal y como existe en profesiones del área de la salud o el derecho. En este contexto, cuestiona la separación entre el saber específico o disciplinar, del saber pedagógico del profesor, entendiendo que el docente es poseedor de un saber que lo distingue de las demás profesiones, en el bien entendido que, para enseñar no es suficiente sólo conocer bien la disciplina, sino que además contar con conocimientos inherentes a la acción de enseñar (12).

Los trabajos referidos al CDC, se han concentrado en investigar la movilización de saberes referidos a la enseñanza, sobre una perspectiva comprensiva de los conocimientos y acciones de los profesores, vistos desde esta mirada como sujetos de esas acciones, sujetos con historia de vida profesional y personal, productores y movilizadores de saberes en el ejercicio de su práctica, y consecuentemente plenos de concepciones sobre el mundo que los rodea, sus estudiantes, los contenidos que les corresponde enseñar, los currículos nacionales, etc.

En este contexto, se pretende indagar acerca de las comprensiones que posee el profesorado de Educación Física (EF) con respecto a la categoría Conocimiento Pedagógico del Contenido (CPC). Por tanto, a objeto de conocer y com- 
prender los componentes del CPC en la disciplina EF. En este contexto, diversos autores $(2,13-16)$ precisan al menos siete componentes del CPC en el área EF. Todos estos aspectos interactúan de manera muy compleja, por tanto no existe la superposición de uno por sobre el otro, sino más bien, una conjunción que se despliega en la práctica y fundamentalmente en la enseñanza de la disciplina Educación Física.

a) El conocimiento de la Educación Física como asignatura: este componente se refiere al conocimiento de los objetivos de la asignatura Educación Física, vale decir los profesores deben conocer el papel y la responsabilidad de la EF como materia escolar, así como la orientación y las características de la EF como disciplina.

b) El conocimiento del plan de estudios de Educación Física: se refiere al conocimiento del currículo nacional y los diferentes enfoques curriculares de la asignatura. Este conocimiento permite a los profesores conocer y comprender la secuenciación de los contenidos en cada grado o nivel escolar.

c) Conocimiento de los métodos de enseñanza en Educación Física: Este componente se refiere al conocimiento de los modelos específicos de enseñanza, estrategias. Comprende las formas como el profesor realiza la enseñanza y la forma como presenta la materia, los modelos de instrucción, demostraciones, explicaciones, analogías, metáforas, ejemplos, tareas de aprendizaje o ejercicios que el profesor utiliza para favorecer que el alumno comprenda un tópico específico de la materia a ser enseñada.

d) El conocimiento del aprendizaje de la Educación Física por parte de los alumnos: En este apartado se trata que el profesor tenga conocimiento acerca de los niveles de desarrollo de los estudiantes y sus habilidades, vale decir los profesores deben conocer las razones de las dificultades de aprendizaje y las fuentes comunes de errores por parte de estos para el aprendizaje de las actividades físicas.

e) Conocimiento curricular del contenido: incluye el conocimiento de los recursos disponibles para estructurar una determinada materia para la enseñanza, así como la articulación horizontal tanto como vertical de los programas de estudio. El conocimiento curricular remite a los conocimientos que permiten al profesor elaborar, adaptar y aplicar propuestas pedagógicas.

f) El conocimiento de la evaluación en Educación Física: este componente consiste en el conocimiento de los principios, características y métodos para evaluar el aprendizaje del estudiante en la EF, de tal manera de implementar aquellas estrategias que sean más pertinentes para evaluar las diferentes dimensiones de la disciplina.

g) El conocimiento de los entornos de enseñanza de la Educación Física: este componente se refiere al conocimiento de los usos y funciones de seguridad en las instalaciones y equipos, así como el conocimiento de la gestión del espacio y los arreglos de los materiales de aprendizaje y herramientas del proceso de enseñanza.

Los componentes anteriormente mencionados, informan a los maestros y formadores de docentes de los tipos de conocimiento que se requiere desarrollar 
en la EF escolar y particularmente en la formación del profesorado. Además, abordar estos componentes desafía a los formadores de docentes a entender y diseñar un marco conceptual que ayude a los futuros profesores no sólo a comprender lo que el CDC significa, sino más bien, cómo aplicar este conocimiento de manera efectiva en el proceso de enseñanza-aprendizaje. En la enseñanza de la EF, el conocimiento pedagógico del contenido es un tipo de conocimiento procesual, que permite al profesor hacer una adaptación del conocimiento declarativo a nivel de comprensión de los alumnos, vale decir hace referencia al entendimiento pedagógico que el profesor posee de los alumnos dentro de un contexto especifico o real de aula (17).

En este sentido, la investigación referida al CDC en el área de la Educación Física plantea que los profesores relevan el conocimiento del contenido o conocimiento de la materia, incluyendo actividades prácticas (atletismo, danza, juegos, gimnasia, actividades al aire libre y de aventura, natación) así como el conocimiento pedagógico como técnicas de enseñanza. Todo lo anterior conlleva a pensar que la EF es una asignatura que enseña "actividad física", cuestión que incluiría entre otros a los deportes. De esta manera los estudiantes deberían saber qué y saber cómo (la comprensión teórica por un lado y la vivencia practica por otro) de las actividades físicas (18-25).

En este contexto, el objetivo de este estudio es describir y categorizar el discurso del profesorado de EF con respecto al Conocimiento Pedagógico del Contenido en el aula de EF y por otro lado, develar las comprensiones subjetivas con que los profesores estructuran y organizan sus estrategias didácticas para favorecer el aprendizaje de los estudiantes.

\section{MATERIAL Y MÉTODOS}

La metodología empleada es de carácter cualitativa, en el bien entendido que esta metodología pretende entender y describir los fenómenos sociales desde el interior (26). En este sentido, hemos optado por una lógica investigativa anclada en el paradigma interpretativo-fenomenológico a partir del que creemos es posible inferir y comprender dichos significados $(27,28)$. Desde este enfoque paradigmático, la metodología cualitativa permite una comprensión subjetiva que emerge a partir de las entrevistas analizadas, entendiendo que estos esconden un contenido educativo caracterizado, implícita y/o explícitamente, por intencionalidades educativas, finalidades, concepciones, exclusiones y valoraciones.

Mediante la entrevista semi-estructurada fue posible indagar en las percepciones que los docentes de EF han construido acerca del CPC. Se realizaron seis entrevistas a profesores las que fueron grabadas en formato audio y transcritas posteriormente siendo fiel al discurso de los participantes

La selección de los participantes fue definida mediante los siguientes criterios:

- Profesores de Educación Física que se encuentren trabajando en colegios de enseñanza básica o media al momento de realizar la investigación. 
- Profesores que trabajen en alguna de las siguientes provincias de la región del Maule: Curicó, Talca, Linares y Cauquenes.

- Profesor titulado con 5 años de experiencia al menos.

- Pleno conocimiento del profesor, para aplicar la entrevista además para ocupar los datos que serán expuestos en nuestra investigación.

- Profesores especialistas de EF tanto de enseñanza básica como de educación media, que trabajan en instituciones educativas con subvención del Estado.

Los procedimientos empleados, en general se ciñeron a las propuestas de fragmentación y articulación de la Grounded Theory (29). El primer paso consistió en hacer visibles las teorías sustantivas de los participantes; para ello se realizó una codificación abierta desde donde emergieron las primeras categorías. Posteriormente se procedió a la agrupación de las categorías secundarias en categorías primarias, realizando una codificación selectiva que permite pasar de la descripción a la interpretación de los datos (26, 30-34). Todos los datos obtenidos en la investigación del apartado cualitativo, se redujeron, codificaron y categorizaron por medio del software de investigación Nvivo 10.

\section{RESULTADOS}

La tabla 1 "Matriz de Sistematización de Categorias", da cuenta del proceso de construcción de categorías, de acuerdo a lo señalado precedentemente. Se presentan las categorías más significativas del estudio, en el bien entendido que una vez definida las categoría previa, comienza el proceso de codificación (33). Las categorías primarias surgen producto de que las categorías secundarias se comienzan a agrupar en diferentes dominios o pequeñas dimensiones que permiten ordenar los datos y darles un sentido teórico. A este proceso Straus y Corbin (34) lo denominan codificación abierta.

\begin{tabular}{|c|c|c|c|}
\hline $\begin{array}{l}\text { CATEGORÍA } \\
\text { PREVIA }\end{array}$ & $\begin{array}{l}\text { CATEGORIA } \\
\text { PRIMARIA }\end{array}$ & CATEGORÍAS SECUNDARIAS & $\begin{array}{c}\text { \% TOTAL DE } \\
\text { REFERENCIAS }\end{array}$ \\
\hline \multirow{6}{*}{$\begin{array}{l}\text { Conocimiento } \\
\text { Pedagógico del } \\
\text { Contenido }\end{array}$} & \multirow{3}{*}{$\begin{array}{l}\text { Conocimiento y } \\
\text { aprendizaje }\end{array}$} & Aprendizaje diferenciado & 5.7 \\
\hline & & Aprendizaje significativo & 19 \\
\hline & & Juego y aprendizaje & 21 \\
\hline & $\begin{array}{l}\text { Conocimiento del } \\
\text { Currículo en EFI }\end{array}$ & Normativa Ministerial & 17.1 \\
\hline & \multirow{2}{*}{$\begin{array}{c}\text { Conocimiento } \\
\text { de Estrategias } \\
\text { Didácticas }\end{array}$} & Didáctica motivadora & 8.6 \\
\hline & & Estrategias diversas & 28.6 \\
\hline
\end{tabular}

Tabla 1. Matriz de sistematización de categorías. 


\section{DISCUSIÓN}

Las entrevistas se analizaron de manera inductiva, lo que permite organizar y categorizar los datos a través del análisis de contenido. El análisis y discusión se ha estructurado de la exposición de los relatos más significados de la categoría secundaria que presenta mayor porcentaje de representatividad de cada una de las categorías primarias.

La identidad de los sujetos entrevistados ha sido protegida con el fin de resguardar su identidad. Los nombres que aquí aparecen son simbólicos y se utilizaron como código del relato.

\section{Categoría Primaria. Conocimiento y Aprendizaje \\ Categoría Secundaria. Juego y Aprendizaje}

Esta categoría posee un porcentaje de frecuencia de $21 \%$ de la totalidad de la muestra de profesores de Educación Física investigados. En ella los profesores investigados relevan el valor del juego como un espacio educativo y de aprendizaje, toda vez que plantean cuestiones como las siguientes;

"Lo que siempre trato de hacer es dar a conocer lo básico del deporte, el cómo se juega, el cómo se practica, reglas básicas para que el niño más que manejarlo al revés y al derecho y que sea un especialista en la materia, tenga la noción de cómo se realiza, las reglas básicas, las ideas generales, partiendo por eso y después de eso solo a través de juego o sea no hacemos entrenamientos de técnicas, no utilizamos una clase completa para practicar un saque bajo en el vóleibol, un saque alto en el vóleibol, se realiza sólo a través de juegos, modificamos de tal manera que el niño jugando valla aprendiendo ciertos gestos de forma hasta natural". Entrevista Joaquín [Cobertura 3,35\%] "Porque igual es bueno enseñar a los niños jugando, igual a través del juego ellos van aprendiendo y la pasan bien y juegos grupales con el balón no se diez pasos punto y luego cambios y así, a ellos les gusta eso. No todo se hace estructurado yo quiero que ellos aprendan jugando, porque los juegos son lo principal aquí son el soporte”. Entrevista Silvio [Cobertura 3,02\%]

"Tenemos una herramienta súper valiosa nosotros en la clase para desarrollar ese tipo de cosas y que los niños a través de los juegos, se trasformen en personas competentes que puedan ser empáticos". Referencia 2 - [Cobertura $1,00 \%]$

El juego es fundamental en el aula de EF, toda vez que comprende dos dominios relevantes para el aprendizaje, el movimiento motor (ejercicio físico) por un lado y el aspecto lúdico por otro. En este sentido, la neurociencia actual ha demostrado que existe una relación directa entre movimiento y aprendizaje y que esta relación se daría durante el transcurso de la vida y por tanto existen actualmente varios programas educativos basados en el juego, preferentemente en la infancia temprana (35). 
En este sentido se puede afirmar que el juego entrega beneficios múltiples e insustituibles ante el más sofisticado aparato tecnológico, pues obliga la mayoría de las veces el contacto con otro, la solución de problemas, el establecimiento de roles, la comunicación, el afecto, la expresión corporal, la ubicación temporoespacial, la relación con los objetos, es decir, conduce a niños y niñas ecológicamente hacia el aprendizaje y el desarrollo socioemocional, mejor que cualquier actividad académica (36).

De acuerdo a Frost, citado en OCDE (35), el juego es esencial para un desarrollo sano, al facilitar la unión del lenguaje con la emoción, el movimiento, la socialización y la cognición. Con respecto a los beneficios del ejercicio podemos mencionar que modela los músculos, el corazón, los pulmones y los huesos, también fortalece los ganglios basales, el cerebelo, el cuerpo calloso, todos ellos zonas claves del cerebro. Por otro lado, desde el punto de vista fisiológico el ejercicio aporta oxígeno al cerebro, pero también neurotropinas (alimento alto en nutrientes) para mejorar el crecimiento y establecer más conexiones entre las neuronas, también el ejercicio de carácter aeróbico ayuda a los procesos de memoria. Por su parte, Pollatschek y Hagen citado en Jensen (37), señalan que los niños que participan en el aula de EF diariamente muestran una mejora motriz, un mejor rendimiento académico y una actitud más favorables hacia la escuela, respecto de sus compañeros que no hacen ejercicio todos los días o que no participan activamente de las sesiones de EF.

En este contexto, el hecho de que los profesores de EF consideren que la premisa "aprender jugando", es relevante a la hora de realizar sus clases, los sitúa en una concepción de aprendizaje vanguardista e innovadora, apoyada por la ingente investigación en neurociencias.

\section{Categoría Primaria. Conocimiento del Currículo en EFI Categoría Secundaria. Normativa Ministerial}

Esta categoría posee un porcentaje de frecuencia de $17.1 \%$ de la totalidad de la muestra de profesores de Educación Física investigados. Los profesores investigados reconocen que, como parte del conocimiento pedagógico del contenido, es menester considerar en su planificación y ejercicio de aula, la normativa emanada desde el Ministerio de Educación.

"Acá en el establecimiento ocupamos planes y programas, porque es un establecimiento Municipal y... nos obligan a pasar los contenidos de los planes y programas. Uno se cuelga un poco del Marco de la Buena Enseñanza" Entrevista Juan [Cobertura 0,37\%]

"Yo creo que a mí me sirve, el Marco de la Buena Enseñanza, a mi gusta arto, pero yo creo que... por algo el Ministerio te está implantando algunas cosas". Entrevista Marco [Cobertura 2,34\%]

"Los planes y programas, me guío por lo que está exigiendo.... la agencia de calidad o la súper intendencia, ellos te dicen, muéstreme su planificación y las 
planificaciones tienen que ir de acuerdo a lo que ellos dicen, es casi impuesto, pero el Marco de la Buena Enseñanza, las Bases Curriculares obviamente las vas ocupando". Referencia 2 - [Cobertura 1,62\%]

En el relato de los profesores investigados se observa que utilizan como documentos de referencia El Marco para la Buena Enseñanza (MBE), las Bases Curriculares y los Programas de Asignatura. El MBE es un documento publicado el año 2003, como parte de la reforma educacional en curso, impulsada por el gobierno del presidente Frei Ruiz Tagle a mediados de los años 90, el texto plantea una perspectiva de desarrollo a lo largo de la vida profesional del maestro, en sus distintas etapas, lo que conlleva a prácticas pedagógicas diferentes, de acuerdo a la experiencia que van adquiriendo los profesores en el transcurso de su vida laboral. El MBE buscó inicialmente, sentar las bases del quehacer profesional docente y especialmente lo que cada profesor y profesora en el país deben saber y saber hacer en el aula, describiendo lo que debe hacer un buen docente. Lo anterior en el bien entendido que los docentes deben asumir un conjunto de responsabilidades, de tal manera de contribuir de manera importante y significativa con el aprendizaje de sus estudiantes. Adicionalmente se busca que sean los propios profesores quienes examinen sus propias prácticas de enseñanza y contrastarlas con parámetros consensuados colectivamente y de esta manera generar procesos de mejora individual y colectivamente (38).

En relación a las Bases Curriculares, la Ley General de Educación (LGE) de 2009 , indica que se deben remplazar las actuales por otras nuevas basadas en Objetivos de Aprendizaje (OA), que definen los objetivos mínimos de aprendizaje que deben alcanzar todos los establecimientos educacionales del país en cada nivel y asignatura. Los programas de estudio del Ministerio de Educación de Chile se construyen a partir de ellos y los establecimientos que optan por programas propios también deben cumplir con los objetivos de aprendizaje de estas bases. Con respecto a Educación Física y Salud, como su nombre lo indica, se orienta a crear buenos hábitos de actividad física y a integrar ésta en la actividad cotidiana de los alumnos. Además, se amplía al logro de actitudes de trabajo en equipo, autocuidado, liderazgo y juego limpio.

\section{Categoría Primaria. Conocimiento de Estrategias Didácticas Categoría Secundaria. Estrategias Diversas}

Esta categoría posee un porcentaje de frecuencia de $28,6 \%$ de la totalidad de la muestra de profesores de Educación Física investigados y es la categoría secundaria con mayor frecuencia.

Los profesores investigados hacen alusión a las distintas maneras con las que es posible abordar el proceso de aprendizaje.

"Se pasa de una actividad a otra con sus descansos, con su hidratación, entonces es la manera que tenemos nosotros acá para funcionar, damos la indicación en la sala, explicamos la actividades, damos a conocer los juegos y 
luego la clase en el patio y hacemos lo que es la parte práctica”. Entrevista Joaquín [Cobertura 1,08\%]

"Los juegos pre deportivos mostramos, en todos los deportes mostramos la historia primero, mostramos las reglas y después un video que vean cómo juegan, los fundamentos básicos y después lo llevamos a la realidad y la etapa de entrenamiento a través de un juego deportivo en el lanzamiento". Entrevista Silvio [Cobertura 5,18\%]

"Mi método es por ejemplo: explicación, demostración, evaluación,... y corrección, siempre a través de los sentidos los niños tiene que ir vivenciando y aprendiendo, por ejemplo mirando como lo hace el profesor, escuchando la explicación, haciéndolo y después corrigiéndolo; siempre siguiente ese orden. Entrevista Manuel [Cobertura 1,61\%]

"Sí, se ocupan muchas analogías, ejemplos, comparaciones; en los que uno saca al niño del contexto técnico de la enseñanza o alguna habilidad y lo lleva a la vida diaria. Ese tipo de comparación o analogía al niño le quedan marcado porque es lo que hace día a día”. Entrevista Marco [Cobertura 1,32\%]

De acuerdo al relato de los sujetos investigados, se comprende que luego de cumplida la fase de planificación de la sesión, corresponde el momento de poner en práctica lo planificado precedentemente, esta instancia es precisamente la clase de EF. No obstante, lo anterior, esta sesión también se encuentra determinada por la problemáticas social, económica y cultural que gira en torno a la escuela, toda vez que el establecimiento educativo no es un espacio aislado y sin relación con su entorno (39).

Con respecto a la sesión, la literatura sugiere que la didáctica empleada por el docente, permita al estudiante vivir un ambiente de plena autonomía, democracia y participación, favoreciendo con ello la formación integral de niños y jóvenes (40). Por otro lado, si bien es cierto la responsabilidad de llevar a cabo la sesión es del profesor(a), no es menos cierto que deba realizar un trabajo aislado del contexto e intereses de los estudiantes, e incluso de carácter colaborativo con los demás profesores de la escuela o, liceo. La propuesta sería asumir con el colectivo de profesores un modelo pedagógico, lo cual supone tener claridad sobre aspectos tales como metas, contenidos, relaciones profesor-alumno, evaluación, concepto de educación y de desarrollo humano entre otros. Por otro lado, en cuanto a las funciones educativas de la escuela y de la educación física, estas tienen que ver con la 'reconstrucción de los conocimientos y las experiencias' que el alumnado tiene en su vida paralela y anterior a la escuela (41), vale decir que no podemos dejar de lado la historicidad del sujeto, en consecuencia, toda vez que desarrollemos una estrategia didáctica en el aula, ésta debe estar en sintonía con quien es el estudiante o los estudiantes a quienes estamos educando, de ahí entonces que la diversidad se convierta en un eje central de las estrategias implementadas al objeto de abordar los intereses y la individualidad de cada uno de ellos.

Para lograr lo anterior, es menester la utilización de diversas estrategias didácticas como las mencionadas por los profesores investigados. Desde esta 
perspectiva los profesores se apoyan en diversos medios para el desarrollo de estrategias didácticas diversas que vayan en función del aprendizaje de todos los estudiantes. La mayor de las dificultades para los docentes está en generar situaciones de aprendizaje que constituyan un problema que el alumno tenga que resolver, y es en el proceso de resolución de la tarea donde se produce el aprendizaje real. La función del docente es la de diseñar y proponer estas situaciones y la de orientar el proceso sin dar soluciones estandarizadas, para así alejarnos, cuando sea posible, de una enseñanza tecnicista de la EF (42).

\section{CONCLUSIONES}

La investigación permitió comprender como el profesorado de EF significa la noción de CPC y la vincula con los aprendizajes que se desarrollan en el aula de Educación Física, a partir del currículo nacional de la disciplina y considerando diferentes métodos y estrategias didácticas. Lo anterior conlleva a que desde su práctica pedagógica los profesores relevan los aspectos asociados a la necesidad de favorecer un aprendizaje diferenciado en su aula, y que se constituya en significativo para los estudiantes, a través de la estrategia del juego como el mejor recurso para generar un conocimiento pertinente y relevante en el área. Por otro lado, las bases curriculares y la normativa ministerial vigente son los documentos que utilizan los profesores para planificar y evaluar sus clases, y consecuentemente enseñar los contenidos que el currículo nacional prescribe.

A la luz de lo expuesto, se desprende que los profesores aspiran a desarrollar una didáctica motivadora, que favorezca el aprendizaje con sus estudiantes, en el bien entendido que la Educación Física es la base de la formación integral del niño, puesto que, por un lado, posee un carácter fuertemente vivencial y por otro, compromete los dominios cognitivo, motor, ético y comunicativo de la persona humana.

En el discurso de los profesores emerge con nitidez la concepción de que el aprendizaje es un proceso complejo y diferenciado, por cuanto no todos los seres humanos aprenden de la misma manera. En el área de la Educación Física la noción de aprendizaje aspira a formar niños y jóvenes que lleguen a ser personas competentes e ilustradas deportivamente, de tal manera que puedan desarrollar habilidades y estrategias que permitan a los jóvenes participar de juegos pre-deportivos y deportivos en el marco de un contexto social y cultural determinado, por otro lado y tan relevante como lo anterior, se pretende generar aprendizajes orientados a la promoción de la salud y la calidad de vida en los distintos periodos etarios

En relación al Conocimiento Pedagógico del Contenido, la investigación nos permite aseverar que un profesor que logra un adecuado dominio de este constructo, es capaz de generar diálogos horizontales en la construcción del conocimiento, favoreciendo instancias pedagógicas que poseen un fuerte acento en lo que los niños traen como capital físico y deportivo desde los propios hogares, 
para a partir de ello co-construir espacios de desarrollo, de convivencia, de juego y participación respetuosos consigo mismo, con el otro y con el entorno. En definitiva, un docente que concibe la disciplina Educación Física como una posibilidad para rescata el valor educativo del deporte, de las expresiones artísticas, del desarrollo motor, del juego, de las actividades en el medio natural, de la danza, el circo, y todo aquello que es posible compartir y desarrollar en el área.

\section{REFERENCIAS BIBLIOGRÁFICAS}

1. Amade-Escot $\mathrm{C}$. The contribution of two research programs on teaching content:"Pedagogical content knowledge" and "didactics of physical education". Journal of Teaching in Physical Education. 2000;20(1):78-101.

2. Graça A. O conhecimento pedagógico do conteúdo: o entendimento entre a pedagogia e a matéria. In: Gomes PBGA, editor. Educação Física e desporto na escola: novos desafios, diferentes soluções Porto: FCDEF-UP; 2001. p. 107-20.

3. Fernández-Balboa J-M, Stiehl J. The generic nature of pedagogical content knowledge among college professors. Teaching and Teacher Education. 1995;11(3):293306.

4. Fenstermacher G. The Knower and the Know: The Nature of Knowledge in Research in Education. Review of Research in Education. 1994.

5. Garritz A, Trinidad-Velasco R. El conocimiento pedagógico del contenido. Educación química. 2004;15(2):98-102.

6. Hashweh* MZ. Teacher pedagogical constructions: a reconfiguration of pedagogical content knowledge. Teachers and Teaching. 2005;11(3):273-92.

7. Marcon D, Nascimento J. Analysis of the pedagogical knowledge of prospective physical education teachers. Kinesiology. 2012;44(2):113-22.

8. Shulman LS. Those who understand: Knowledge growth in teaching. Educational researcher. 1986;15(2):4-14.

9. Shulman L. Knowledge and teaching: Foundations of the new reform. Harvard educational review. 1987;57(1):1-23.

10. An S, Kulm G, Wu Z. The pedagogical content knowledge of middle school, mathematics teachers in China and the US. Journal of mathematics teacher education. 2004;7(2):145-72.

11. Shulman LS. Conocimiento y enseñanza: fundamentos de la nueva reforma. Profesorado Revista de Currículum y formación de Profesorado. 2005;9(2):0.

12. Gaia S, Cesário M, Tancredi RMSP. Formação profissional e pessoal: a trajetória de vida de Shulman e suas contribuições para o campo educacional. Revista Eletrônica de Educação. 2007;1(1):142-55.

13. Armour KM, Yelling M. Professional development and professional learning: Bridging the gap for experienced physical education teachers. European Physical Education Review. 2004;10(1):71-93.

14. Fernández-Balboa J-M. Knowledge base in physical education teacher education: $A$ proposal for a new era. Quest. 1997;49(2):161-81. 
15. Griffin L, Dodds P, Rovegno I. Pedagogical content knowledge for teachers: Integrate everything you know to help students learn. Journal of Physical Education, Recreation \& Dance. 1996;67(9):58-61.

16. McCaughtry N. Elaborating pedagogical content knowledge: What it means to know students and think about teaching. Teachers and teaching. 2005;11(4):379-95.

17. Iserbyt $P$, Ward $P$, Li W. Effects of improved content knowledge on pedagogical content knowledge and student performance in physical education. Physical Education and Sport Pedagogy. 2017;22(1):71-88.

18. Almonacid-Fierro A, Feu S, Carrizosa MV. Validación de un cuestionario para medir el Conocimiento Didáctico del Contenido en el profesorado de Educación Física. Retos. 2018(34):132-7.

19. Ayvazo S, Ward P. Pedagogical content knowledge of experienced teachers in physical education: Functional analysis of adaptations. Research Quarterly for Exercise and Sport. 2011;82(4):675-84.

20. Castejon Oliva FJ, Giménez Fuentes-Guerra FJ. Conocimiento del contenido y conocimiento pedagógico del contenido de educación física en educación secundaria. RETOS Nuevas Tendencias en Educación Física, Deporte y Recreación. 2017(32).

21. Iserbyt $P$, Ward $P$, Martens J. The influence of content knowledge on teaching and learning in traditional and sport education contexts: an exploratory study. Physical Education and Sport Pedagogy. 2016;21(5):539-56.

22. MacPhail A, Tannehill D, Karp GG. Preparing physical education preservice teachers to design instructionally aligned lessons through constructivist pedagogical practices. Teaching and Teacher Education. 2013;33:100-12.

23. You J. Portraying physical education-pedagogical content knowledge for the professional learning of physical educators. Physical Educator. 2011;68(2):98.

24. Ward P, Ayvazo S. Pedagogical content knowledge: Conceptions and findings in physical education. Journal of Teaching in Physical Education. 2016;35(3):194-207.

25. Ward P, Kim I, Ko B, Li W. Effects of improving teachers' content knowledge on teaching and student learning in physical education. Research quarterly for exercise and sport. 2015;86(2):130-9.

26. Gibbs G. El análisis de datos en investigación cualitativa: Ediciones Morata; 2012.

27. Canales M. Metodologías de investigación social. Introducción a los oficios. Santiago: Editorial LOM; 2006.

28. Ruiz J. Metodología de la investigación cualitativa. Bilbao: Editorial Universidad de Deusto; 2003.

29. Glaser BG, Strauss AL, Strutzel E. The discovery of grounded theory; strategies for qualitative research. Nursing research. 1967;17(4):364.

30. Flores R. Observando observadores: una introducción a las técnicas cualitativas de investigación social: Ediciones UC; 2009.

31. Kvale S. Las entrevistas en investigación cualitativa: Ediciones Morata; 2011.

32. Taylor S, Bogdan R. Introducción a los métodos cualitativos de investigación. Barcelona: Paidós 1984.

33. Coffey A, Atkinson P. Encontrar el sentido a los datos cualitativos: estrategias complementarias de investigacion: Editorial Universidad de Antioquia; 2003. 
34. Strauss AL, Corbin J, Zimmerman E. Bases de la investigación cualitativa: técnicas y procedimientos para desarrollar la teoría fundamentada: Universidad de Antioquia Medellín; 2002.

35. OCDE. La comprensión del cerebro: El nacimiento de una ciencia del aprendizaje. Santiago: Universidad Catolica Cardenal Raul Silva Henriquez; 2009.

36. Oyarce $M$, Almonacid $A$. Cognición, juego y aprendizaje: una propuesta para el área de la primera infancia. Revista Infancia, Educación y Aprendizaje. 2015;1(1):162-77.

37. Jensen E. Cerebro y aprendizaje: competencias e implicaciones educativas. Madrid: Narcea; 2010.

38. Mineduc C. Marco para la buena enseñanza. Santiago de Chile: C\&C impresores. 2003.

39. Seners P. La lección de Educación Física. Barcelona: INDE; 1995.

40. Castro-Carvajal JA, Martinez-Escudero LJ, Chaverra-Fernández BE. La investigación en pedagogía y didáctica aplicada a la educación física. Educación y educadores. 2012;15(3).

41. Moreno A, Marlen C, Almonacid A, Vargas A. La educación física chilena. Un modelo tecnocrático de la enseñanza y desvalorización del colectivo docente. Tandem Didáctica de la Educación Física. 2013;42:7-17.

42. Lagardera F, Masciano A. Un rayo de luz en el lado oculto de la educación física. La pedagogía de las conductas motrices en el gimnasio Olimpia de Chivilcoy. Revista Iberoamericana de Ciencias de la Actividad Física y el Deporte. 2014;3(1):31-45.

\section{$N^{\circ}$ Referencias totales: 42 \\ $N^{\circ}$ Referencias Revista: 1}

